\title{
Generating Questions for Inquiry-Based Learning of History in Elementary Schools by Using Stereoscopic 3D Images
}

\author{
Takashi SHIBATA $^{\dagger \text { a) }}$, Kazunori SATO ${ }^{\dagger \dagger, \dagger \dagger}$, and Ryohei IKEJIRI ${ }^{\dagger \dagger \dagger}$, Nonmembers
}

\begin{abstract}
SUMMARY We conducted experimental classes in an elementary school to examine how the advantages of using stereoscopic 3D images could be applied in education. More specifically, we selected a unit of the Tumulus period in Japan for sixth-graders as the source of our 3D educational materials. This unit represents part of the coursework for the topic of Japanese history. The educational materials used in our study included stereoscopic 3D images for examining the stone chambers and Haniwa (i.e., terracotta clay figures) of the Tumulus period. The results of our experimental class showed that $3 \mathrm{D}$ educational materials helped students focus on specific parts in images such as attached objects of the Haniwa and also understand 3D spaces and concavo-convex shapes. The experimental class revealed that 3D educational materials also helped students come up with novel questions regarding attached objects of the Haniwa, and Haniwa's spatial balance and spatial alignment. The results suggest that the educational use of stereoscopic 3D images is worthwhile in that they lead to question and hypothesis generation and an inquiry-based learning approach to history.

key words: stereoscopic images, education, school, 3D display, historical learning
\end{abstract}

\section{Introduction}

In recent years, there have been rapid developments in the technology involved in presenting images on displays. Besides being incorporated into everyday activities, display technologies promote the use of information and communication technology (ICT) in education. For instance, tablets have become increasingly popular in elementary school classrooms as a means of improving educational quality. In Japan, the Ministry of Education, Culture, Sports, Science and Technology (MEXT) continues to actively promote the use of ICT in education through large-scale projects that investigate the educational effectiveness of tablets and other educational equipment; such projects involve researchers conducting practice classes [1].

In this way, teachers and students in school have started to use new display technologies and related technologies, such as touchscreens and digital pens. Promoting ICT in education may allow students to get familiar with digital con-

\footnotetext{
Manuscript received March 7, 2017.

Manuscript revised June 8, 2017.

${ }^{\dagger}$ The author is with Tokyo University of Social Welfare, Isesaki-shi, 372-0831 Japan.

${ }^{\dagger}$ The author is with Tokoha University, Shizuoka-shi, 4200911 Japan.

${ }^{\dagger}$ The author is with Tohoku University, Sendai-shi, 980-8579 Japan.

${ }^{\dagger+\dagger}$ The author is with The University of Tokyo, Tokyo, 113-0033 Japan.

a) E-mail: tashibat@ed.tokyo-fukushi.ac.jp

DOI: 10.1587/transele.E100.C.1012
}

tents, new displays, and new media such as stereoscopic 3D images, virtual reality (VR), and augmented reality (AR). In addition to the investigation of the educational effects of ICT, research on the functionality of new image technology is needed in the field of education.

Recent display technologies enable the presentation of stereoscopic 3D images through more diverse media such as televisions, mobile devices, and cinema screens. There are increasing opportunities for people to view stereoscopic $3 \mathrm{D}$ content. In addition, there are more opportunities to make stereoscopic 3D content because of the availability of consumer 3D cameras in the market. It is therefore becoming increasingly possible to use stereoscopic 3D images in school education. Some school teachers have started using 3D expressions in educational materials through stereo-pair images and anaglyph 3D images. By using recent technologies, the innate utility of 3D expressions can be utilized in school education more effectively.

In school education, the quality of learning is of primary importance. Therefore, there is little use for new technologies unless they promote students' learning. In other words, it is important to consider how a new technology affects students' learning in comparison with conventional educational methods and materials.

In this study, we conducted experimental classes at an elementary school and evaluated the educational effects of using stereoscopic 3D images on a 3D television. In particular, we aimed to clarify the differences in the outcomes when using 3D educational content as opposed to using conventional $2 \mathrm{D}$ content. We also aimed to maximize the advantages of 3D images to improve educational quality and students' learning.

\section{Stereoscopic 3D Images in Education}

\subsection{Effectiveness of Stereo Viewing in School}

There are numerous studies related to the effectiveness of stereoscopic 3D images in mainly scientific visualization [2], [3] and medical applications [4], [5]. For example, Aitsiselmi and Holliman reported that stereo viewing on 3D displays was effective for completing a mental rotation task, a cognitive process that involves the mental rotation of objects [6]. However, there has been little research conducted on school education apart from some case studies. Bamford, for example, explored the effectiveness of using 3D images in the classroom and its potential as a teaching and learning 
tool [7]. Bamford showed that students respond positively to visual stimuli and suggested that 3D images could increase student motivation and engagement. Shibata reported that in formal education, students are interested in learning, and the use of stereo 3D movies and 3D educational materials could provide them with a strong sense of depth in their learning [8]. In Shibata's study, approximately $90 \%$ of the students who participated looked forward to attending another class employing 3D educational content. This suggests that 3D images could be an effective learning tool for inducing student interest. In addition, Shibata et al. reported that 3D educational materials could help students focus on specific details and understand 3D spaces or concavo-convex shapes [9].

\subsection{Inquiry-Based Learning of and Generated Questions Related to History}

Inquiry-based learning is increasingly being emphasized as an effective method of educational practice. For example, the Japanese government's teaching guidelines for elementary school, junior high school, and high school require inquiry-based learning in social studies and history [10][12].

Collaborative inquiry-based learning is mainly divided into eight processes: (1) orienting and asking questions, (2) hypothesis generation, (3) planning, (4) investigation, (5) analysis and interpretation, (6) model conclusions and evaluations, (7) communication, and (8) prediction [13]. Therefore, it is important as a first step to promote the generation of additional and deeper questions by students themselves. This is especially important as the subsequent learning activity depends on the generation of questions. In this study, we conducted the first two steps as an inquiry-based learning process in an experimental class at an elementary school.

Additionally, the generation of questions has received attention from scholars of historical education. Many researchers insist that simply memorizing historical facts is insufficient and that it is more important to foster historical reasoning (also referred to as "historical thinking" or "historical literacy") [14] and to implement collaborative inquiry into the study of history [15]. There are six components of historical reasoning: asking historical questions, using sources, implementing contextualization, engaging in argumentation, using substantive concepts, and employing meta-concepts. Asking historical questions may function as the engine for historical reasoning [14]. Therefore, question generation is one of the essential competencies for historical learning, together with inquiry-based learning.

In addition, using educational materials that provide rich information could also stimulate question generation. For example, McCall reported that junior high school students generated an impressive range of deep questions when using a simulation game [16]. Therefore, stereoscopic 3D images might also have a positive effect on generating questions in inquiry-based learning of history.

\subsection{Human Factors Aspect}

Since 2005, when 3D movies and theaters were introduced, public concern regarding potential adverse effects associated with prolonged viewing of 3D images has increased. Although there are many potential causes of visual discomfort when viewing stereoscopic 3D images, the vergenceaccommodation conflict remains a key problem with stereo 3D displays because it occurs in all conventional stereo 3D displays [17], [18]. Therefore, it is of critical importance to consider how 3D images can be presented as safely as possible, especially in classrooms.

For their use in elementary school social studies classes, we considered the fact that stereoscopic 3D images are generally viewed at a different size on 3D displays and with different viewing distances in a classroom [9] by children with smaller interpupillary distances than those of adults. People who have a small interpupillary distance perforce view more emphasized stereo images than those who have a larger interpupillary distance when viewing the same stereo images on a 3D display of the same size. In creating 3D educational materials and setting up 3D displays in the classroom, we intended to make 3D viewing as comfortable as possible [18]-[20]. More specifically, we set the uncrossed disparity (i.e., stereo images behind the screen) and crossed disparity (i.e., stereo images in front of the screen) in the images to be less than one degree within the classroom as an index of comfort. In order to avoid a large disparity, we especially considered that students in the classroom sometimes viewed the screen with a short viewing distance. In addition, we excluded possible severe adverse factors such as misalignments of the right and left images and frame violations.

\section{Purpose}

Stereoscopic 3D images can directly express depth, concavity, and convexity and therefore can provide rich information compared with that provided by conventional 2D educational materials. In this study, we hypothesized that stereoscopic 3D images could help students generate more questions and come up with novel questions that could not be generated from viewing 2D images. As described above, the generation of questions by students themselves is the first step of inquiry-based learning and hence has an important role in school education.

The purpose of the experimental class in this study was to evaluate the advantages of using $3 \mathrm{D}$ images in the inquiry-based learning of history. We focused on the questions generated by students with the help of the provided educational materials. 


\section{Methods}

\subsection{Participants}

We conducted an experimental class for sixth graders at a public elementary school in Japan. Students from two classes (Class 1: 26 students; Class 2: 30 students) participated in the study. All of the students who attended a class that featured the 3D viewing had normal stereoacuity according to a self-report of the stereo viewing test completed before the class took place. Those who normally wore optical correction continued to wear them during the experimental class.

\subsection{Learning Content}

In this study, we aimed to utilize and evaluate stereoscopic 3D images in an actual school classroom. Therefore, we needed to choose the appropriate educational unit and make the 3D educational materials accordingly before conducting an experimental class. Our basic concept for 3D educational materials was that the students should view content in 3D if it is appropriate for stereo viewing.

After a discussion about the appropriate educational unit for evaluating 3D effects, a unit of the Tumulus period in Japan, which represents part of the Japanese history coursework from the sixth-grade curriculum, was selected as the learning content. From the third to the seventh centuries, many tumuli, or Kofun in Japanese, were built across Japan. Kofun were the burial mounds of the ruling elite, with monumental structures indicating powerful political status (Fig. 1). The mounds contained large stone chambers with terracotta clay figures called Haniwa, which were arranged on and around the mounds.

The elementary students' key learning objectives included having an understanding of the features of the Tumulus period by studying the large burial mounds (Kofun), stone chambers (Fig. 2), and the exquisite terracotta clay figures (Haniwa) as shown in Fig. 3. We selected this unit because the stone chambers and the Haniwa have complicated forms and figures, and we hypothesized that it would be difficult for the students to understand such complicated shapes from conventional textbooks or 2D images. In other words, we deemed this unit to be appropriate for assessing the functionalities of stereoscopic 3D images.

\subsection{Procedure}

The experimental class was conducted after the students had already attended regular classes on the Tumulus period. The two regular classes were conducted by the same teacher in order to teach the same learning content. Figure 4 shows a flow of the classes. In the experimental class, two movies on the subject of the Kofun, including stone chambers and Haniwa, as shown in Figs. 1, 2, and 3, were used. The content on the Kofun consisted of 14 scenes, and the duration

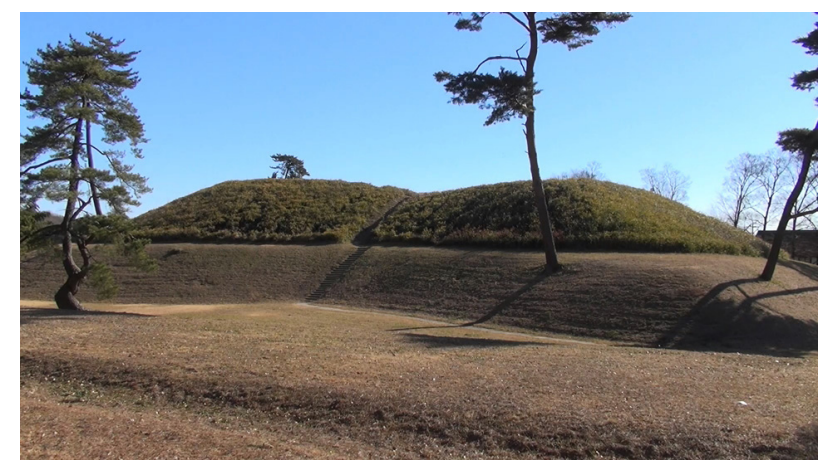

Fig. 1 Tumuli (i.e., Kofun) were the burial mounds of the ruling elite, and built from the third to the seventh centuries across Japan.

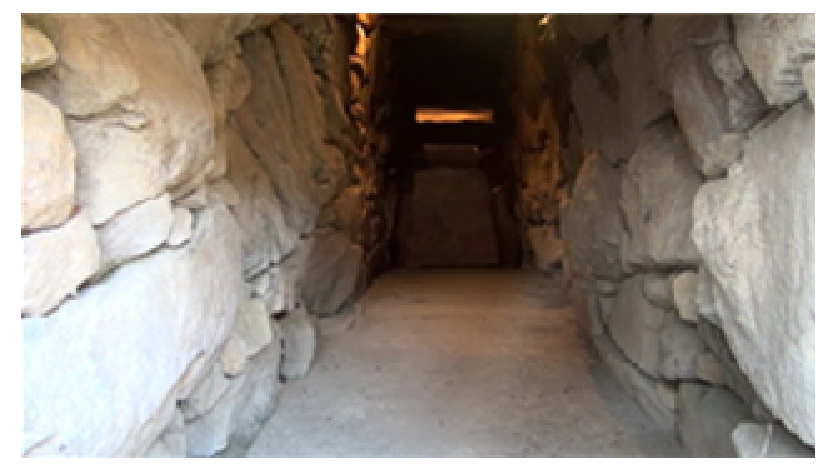

Fig. 2 A passage from the entrance that leads to the stone chamber in a burial mound (Kofun) captured through educational materials.

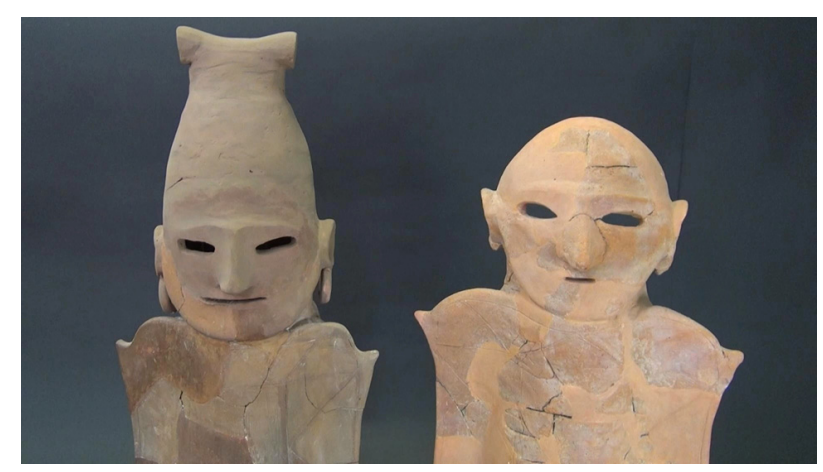

Fig. 3 Exquisite terracotta clay figures (Haniwa) that were arranged on and around the mounds.

of the movie was $1 \mathrm{~min} 47 \mathrm{~s}$. The Haniwa content consisted of 15 scenes, and the duration of the movie was $1 \min 54 \mathrm{~s}$. The viewing time for each movie shown in the class was 5 min because each movie was repeated for $5 \mathrm{~min}$. The experimental group that used the stereoscopic 3D movie was randomly assigned from two 6th-grade classes, and we called it Class 1. Therefore, the students in Class 1 viewed the movies in 3D, whereas those in Class 2 viewed them in 2D. Before viewing the movies, the students were instructed to write out their questions about the Kofun, the Haniwa, and any other related topic after the movie observation.

The display was a 49-inch 3D television with polarized 
glasses (KD-49X8500B, Sony), placed in front of the classroom's blackboard (Fig. 5). The actual size of the screen was $108 \mathrm{~cm}$ horizontally and $61 \mathrm{~cm}$ vertically. The viewing distance of the students in the front and last rows was in the range of $1.5 \mathrm{~m}$ to $6 \mathrm{~m}$ approximately. The 3D movies were prepared in a Multiview Video Coding format. The left view of the stereoscopic 3D movies was used to conduct for the 2D observation.

After the viewing of each of the two movies, the students completed a worksheet that encouraged them to write out their questions about the Kofun, the Haniwa, and anything else that might have emerged from viewing the educational materials. The students made a list of as many questions as possible in $3 \mathrm{~min}$. As a pre-test for the study, the students were also asked to complete an identical worksheet in $6 \mathrm{~min}$ at the end of their previous regular classes.

\section{Regular class on the Tumulus period (45 min)}

1. Regular learning using text book etc.

2. Pre-test to generate questions (6 min)

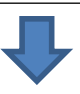

\begin{tabular}{|l|}
\hline \multicolumn{1}{|c|}{ Experimental class (45 min) } \\
\hline 1. Review of previous lesson and instruction \\
2. 3D or 2D observation (Kofun content) (5 min) \\
Experimental group in 3D \& Control group in 2D \\
3. Post-test 1 to generate questions ( 3 min) \\
4. 3D or 2D observation (Haniwa content) (5 min) \\
Experimental group in 3D \& Control group in 2D \\
5. Post-test 2 to generate questions ( 3 min) \\
6. Group discussion and hypothesis generation \\
\hline
\end{tabular}

Fig. 4 Flow of the experimental classes in the study.

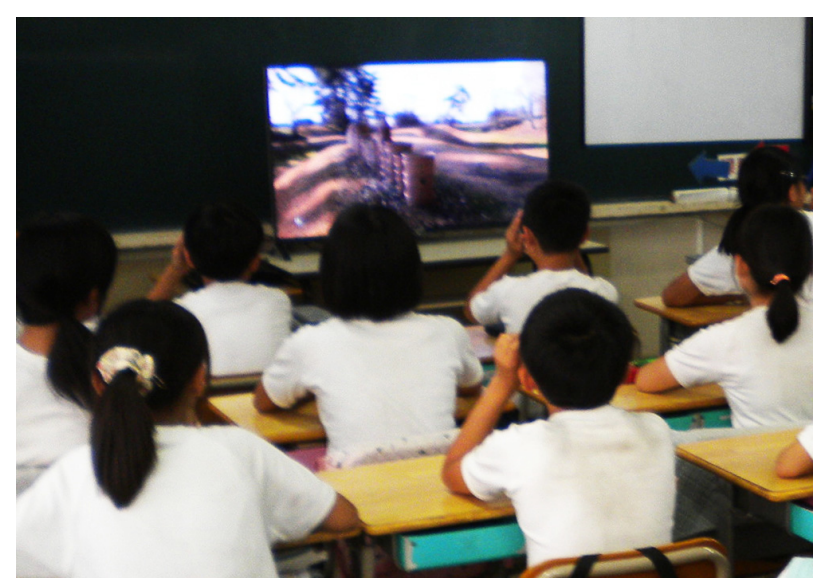

Fig.5 A scene from the experimental 3D classroom in an elementary school.
In the experimental class, the students discussed their hypotheses about the questions in a small group after completing the two worksheets, which is the second step of inquiry-based learning.

\subsection{Analysis}

For our analysis, we focused on two evaluation items: the number of questions and the differences in the categories of questions generated after 3D and 2D viewings. Regarding the number of questions, a greater number of questions can be interpreted as indicating that the educational materials were more effective because the students could actually generate questions, which is the first step of conducting inquiry-based learning. Regarding the differences in the categories of questions, the category which was generated only from $3 \mathrm{D}$ viewing can be interpreted as a characteristic educational effect of 3D educational materials.

\section{Results}

\subsection{Number of Questions}

We compared the results of the pre-test and the two posttests, which were of the same duration (6 min) and used to generate questions. The average pre-test numbers were 5.13 in $2 \mathrm{D}$ and 4.77 in $3 \mathrm{D}$, respectively, and the average posttest numbers were 7.63 in $2 \mathrm{D}$ and 9.54 in 3D, respectively. Figure 6 illustrates the results for the number of questions the students generated for both the 2D and 3D observations before and after they had viewed the educational materials. The scores were averaged on the basis of the number of students in each class. The error bars represent the standard errors of the scores.

A mixed-design analysis of variance (ANOVA) was performed to explore an interaction between educational materials (2D vs. 3D; between subjects) and measuring time (pre-test vs. post-test; within subjects). There was a statistically significant main effect of measuring time $(F(1,54)=$ $85.27, p<.001)$, and a significant interaction with educational materials and measuring time $(F(1,54)=8.31$, $p<.01)$. Focusing on the results of the 3D images, the

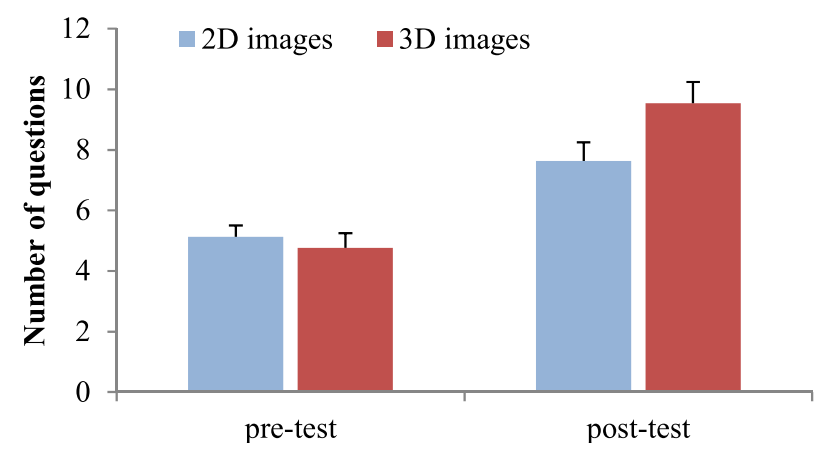

Fig. 6 Changes in the number of questions generated before and after viewing the given educational materials. 
Table 1 Differences in the categories of questions about the Kofun content ( $\mathrm{N}$ means the number of students generating questions belonging to the category).

\begin{tabular}{|c|c|c|c|c|c|c|}
\hline \multicolumn{2}{|l|}{ Categories in the 3D only group } & \multicolumn{3}{|c|}{ Categories in both the 3D and 2D groups } & \multicolumn{2}{|l|}{ Categories in the $2 \mathrm{D}$ only group } \\
\hline Category & $\mathrm{N}$ & Category & $N(3 D)$ & $\mathrm{N}(2 \mathrm{D})$ & Category & $\mathrm{N}$ \\
\hline Why was there a groove around the Kofun? & 2 & Why do the trees stand on top of the Kofun? & 11 & 2 & $\begin{array}{l}\text { Why was the entrance of the Kofun at the } \\
\text { side? }\end{array}$ & 9 \\
\hline Why was the size of the Haniwa different? & 2 & Why was the wall made of stone? & 4 & 4 & Why were the Kofun high? & 4 \\
\hline Why were the Haniwa not placed in order? & 2 & Why were the Kofun made with soil piled on? & 3 & 5 & Why did they need to make the Kofun? & 2 \\
\hline Why were the Haniwa black? & 2 & Why were the Kofun large? & 3 & 4 & How many entrances were there? & 2 \\
\hline Where would the dead leave for? & 2 & Why did the rocks not fall? & 3 & 4 & Why was the inside of the Kofun not dark? & 2 \\
\hline Why did people enter the stone chamber? & 2 & Why were the Haniwa placed? & 2 & 5 & $\begin{array}{l}\text { Why was the inside of the Kofun made of } \\
\text { stone? }\end{array}$ & 2 \\
\hline \multirow[t]{6}{*}{ Where were the rocks brought from? } & 2 & Why were many Haniwa placed? & 2 & 5 & Why was the wall firm? & 2 \\
\hline & & $\begin{array}{l}\text { Why were the Haniwa placed in the stone } \\
\text { chamber? }\end{array}$ & 2 & 5 & \multirow{5}{*}{$\begin{array}{l}\text { Why were the Haniwa which looked like the } \\
\text { dishes placed there? } \\
\text { Why were the powerful families buried in the } \\
\text { basement? }\end{array}$} & 2 \\
\hline & & $\begin{array}{l}\text { Why were the dead buried in the heart of the } \\
\text { stone chamber? }\end{array}$ & 2 & 4 & & 2 \\
\hline & & Why were the Haniwa placed in the passage? & 2 & 3 & & \\
\hline & & $\begin{array}{l}\text { Why was there a variety of forms in the } \\
\text { Kofun? }\end{array}$ & 2 & 2 & & \\
\hline & & Why were there many entrances? & 2 & 2 & & \\
\hline
\end{tabular}

Table 2 Differences in the Categories of questions about the Haniwa content ( $\mathrm{N}$ means the number of students generating questions belonging to the category).

\begin{tabular}{|c|c|c|c|c|c|c|}
\hline \multicolumn{2}{|l|}{ Categories in the 3D only group } & \multicolumn{3}{|c|}{ Categories in both the 3D and 2D groups } & \multicolumn{2}{|l|}{ Categories in the $2 \mathrm{D}$ only group } \\
\hline Category & $\mathrm{N}$ & Category & $\mathrm{N}(3 \mathrm{D})$ & $N(2 D)$ & Category & $\mathrm{N}$ \\
\hline $\begin{array}{l}\text { Why were the figures of animals attached to } \\
\text { the Haniwa? }\end{array}$ & 8 & Why were there many kinds of Haniwa? & 11 & 11 & Why were there Haniwa around the Kofun? & 6 \\
\hline In what order were the Haniwa placed? & 5 & Why were many Haniwa placed? & 4 & 5 & Why were there the Haniwa of warriors? & 2 \\
\hline $\begin{array}{l}\text { Why were the ornaments attached to the } \\
\text { Haniwa? }\end{array}$ & 3 & Why were there Haniwa? & 4 & 4 & Why were many cylindrical Haniwa placed? & 2 \\
\hline Why were the Haniwa lined up in a row? & 2 & Why was the hole opened in the Haniwa? & 3 & 5 & Why were the Haniwa placed irregularly? & 2 \\
\hline $\begin{array}{l}\text { Was there a limit on the number of Haniwa } \\
\text { that could be placed? }\end{array}$ & 2 & Why were the Haniwa placed? & 2 & 6 & Why were the Haniwa not flat? & 2 \\
\hline What did the variations of the Haniwa mean? & 2 & How many kinds of Haniwa were there? & 5 & 2 & $\begin{array}{l}\text { Why was the expression of the Haniwa } \\
\text { similar? }\end{array}$ & 2 \\
\hline How large were the Haniwa? & 2 & Why were the Haniwa placed all in one piece? & 3 & 2 & Why were the Haniwa of warriors large? & 2 \\
\hline \multirow[t]{4}{*}{ Why were the clay figures called Haniwa? } & 2 & $\begin{array}{l}\text { How many Haniwa were there around a } \\
\text { Kofun? }\end{array}$ & 3 & 2 & & \\
\hline & & Why was the size of the Haniwa different? & 3 & 2 & & \\
\hline & & Why were the Haniwa aligned? & 2 & 3 & & \\
\hline & & Who made the Haniwa? & 2 & 2 & & \\
\hline
\end{tabular}

number of questions that were generated in the post-test was significantly higher than those in the pre-test. Furthermore, the increased extent was greater than in the case of 2D images.

\subsection{Differences in the Categories of Questions}

Table 1 presents the categories of questions for the Kofun content and Table 2 presents the categories of questions for the Haniwa content. The tables are divided into three columns: categories that were shown to the 3D only group, categories showed to both the $3 \mathrm{D}$ and $2 \mathrm{D}$ groups, and categories showed to the 2D only group.

The process to generate categories was as follows:
First, a category was generated if more than two students from each group had generated similar questions. Second, the categories generated from the first step were checked with the category of the 3D only group, the 3D and 2D group, and the 2D only group. The analytical work was implemented by one of authors in the first instance. Then, to ensure the reliability of the categorization, another author checked whether the categorization was valid for all categories and whether all of the questions had been derived from the students. After this checking process, the two authors discussed the points that were put forth by the second author. As a result, the categorization for 26 out of the 479 questions was discussed and the categorization for 21 ques- 
tions was modified on the basis of this discussion.

First, we focused on the results of the 3D only group. For the Kofun content, the categories of questions generated for the 3D only group were seven (the left column of Table 1). These questions included "Why was there a groove around the Kofun?", "Why was the size of the Haniwa different?", "Why were the Haniwa not placed in order?", "Why were the Haniwa black?", "Where would the dead leave for?", "Why did people enter the stone chamber?", and "Where were the rocks brought from?" For example, the questions generated by the students in the first category of "Why was there a groove around the Kofun?" were "Why was there a groove?" and "Why was the area around the Kofun dented?"

For the Haniwa content, nine categories of questions were generated in 3D only group (left column of Table 2): "Why were the figures of animals attached to the Haniwa?", "Why were the Haniwa standing without falling?", "In what order were the Haniwa placed?", "Why were the ornaments attached to the Haniwa?", "Why were the Haniwa lined up in a row?", "Was there a limit on the number of Haniwa that could be placed?", "What did the variations of the Haniwa mean?", "How large were the Haniwa?", and "Why were the clay figures called Haniwa?". For example, the first category of "Why were the figures of animal attached to the Haniwa?" included the questions "Why were the figures of the human and the animal attached to the cylindrical Haniwa?" and "Why were the figures of animals attached to the Haniwa?" Additionally, the second category of "Why were the Haniwa standing without falling?" included the questions "Why were the Haniwa not falling?" and "Why were the Haniwa standing without falling?"

Second, we focused on the results of the characteristic categories in both the 3D only and 2D only groups. We defined that a category with three or more questions was a characteristic response to that group in order to eliminate eventuality and improve reliability. This is because the number 2 is the smallest value to make a category. In the $3 \mathrm{D}$ only group, the category with the largest number was "Why were the figures of animals attached to the Haniwa?" $(\mathrm{N}=8)$ from the Haniwa content. These questions were focused on the animal shaped objects attached to the side of the Haniwa. The second category was "Why were the Haniwa standing without falling?" $(\mathrm{N}=5)$ from the Haniwa content. These questions were focused on the spatial balance that the Haniwa does not collapse. The third category was "In what order were the Haniwa placed?" $(\mathrm{N}=5)$ from the Haniwa content. These questions were focused on the order in which the Haniwa are lining up (i.e. spatial alignment). The fourth category was "Why were the ornaments attached to the Haniwa?" ( $\mathrm{N}=3$ ) from the Haniwa content. These questions, like the first category, were focused on the objects attached to the side of the Haniwa.

In the 2D only group, the category with the largest number was "Why was the entrance of the Kofun at the side?" ( $\mathrm{N}=9)$ from the Kofun content. These questions were focused on the overall positional relationship that the entrance to the stone chamber laid on the side of the Kofun. The second category was "Why were there Haniwa around the Kofun?" ( $\mathrm{N}=6)$ from the Haniwa content. These questions were focused on the overall positional relationship as well as the first category. The third category was "Why were the Kofun high?" ( $\mathrm{N}=4)$ from the Kofun content. These questions were focused on the overall characteristics of the Kofun.

\subsection{Hypothesis Generation from Questions}

Next, we present how the category of questions generated in the 3D only group led to the following step of inquiry-based learning, namely, hypothesis generation. For example, one of the 3D viewing groups that selected the question "Why were the figures of animals attached to the Haniwa?" (the first category of questions for the Haniwa content) generated nine hypotheses: "Because the human who was buried liked animals," "Because they thought of cylindrical Haniwa as a tree," "Because animals were luxury foods," "Because many people liked animals," "Because the animal was kept by the human who was buried," "Because animals looked delicious," "Because there were animal deities," "Because they drew the scene of the human being chased by the animal," and "Because the human was being helped by the animal."

Another 3D viewing group that selected the question "Why were the Haniwa not falling?" (the second category of questions for the Haniwa content) generated four hypotheses: "Because the Haniwa were buried in the soil," "Because the balance of the Haniwa was correctly calculated," "Because the Haniwa were made in order not to be blown by passing wind," and "Because the Haniwa created were heavy."

\section{Discussions}

Our results showed that the number of student questions after viewing 3D educational materials was significantly higher than that during the pre-test compared to 2D educational materials. Note that the students had already attended the regular class before the experimental class. In other words, the student had finished normal learning in class and understood the learning content before the experimental class. Therefore, the 3D educational materials were effective in that the students were able to generate more questions and think deeply about the learning content.

To clarify the effects of the 3D educational materials, we performed a qualitative analysis of the questions generated by the students. From the results of the Kofun content (Table 1), in the 3D only group, there were several questions mainly about the size, order, and color of the Haniwa. On the other hand, in the 2D only group, there were questions about the overall positional relationship, such as the positional relationship between the Haniwa and the entrance to the stone chamber, and the height of the Haniwa. Thus, it was suggested that $3 \mathrm{D}$ educational materials have an effect to make specific parts attractive. 
Focus areas when viewing 3D educational materials

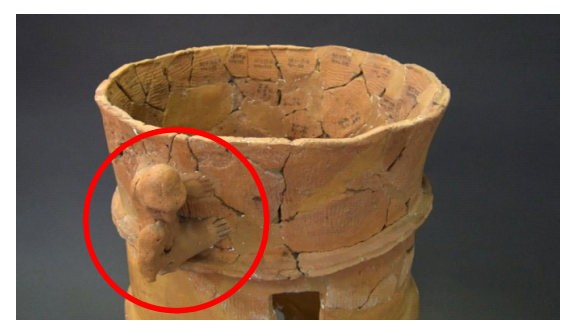

Attached objects of Haniwa

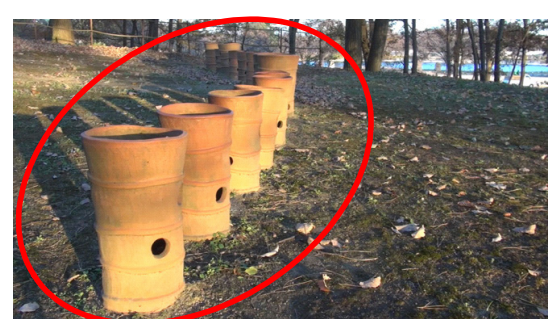

Spatial balance of Haniwa

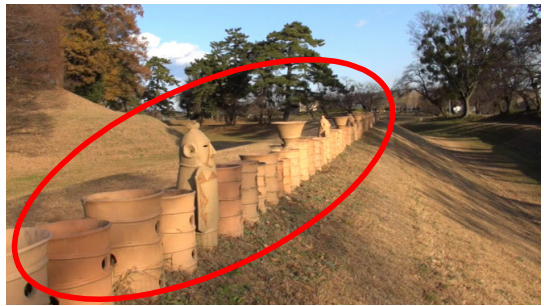

Spatial alignment of Haniwa

Focus areas when viewing 2D educational materials

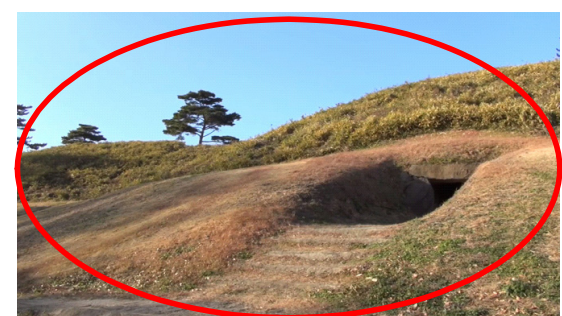

Position of Kofun and the entrance to the stone chamber

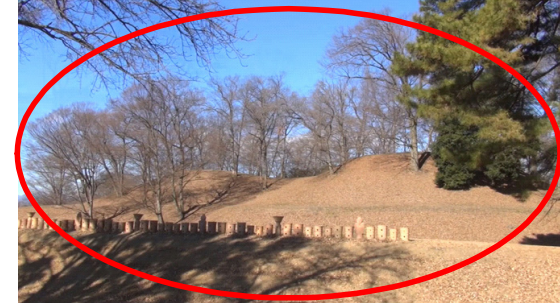

Arranged Haniwa around Kofun

Fig. 7 Differential focus areas of the students' attention from the viewings of the 3D and 2D educational materials and the related scenes that the students seemed to have referred to.
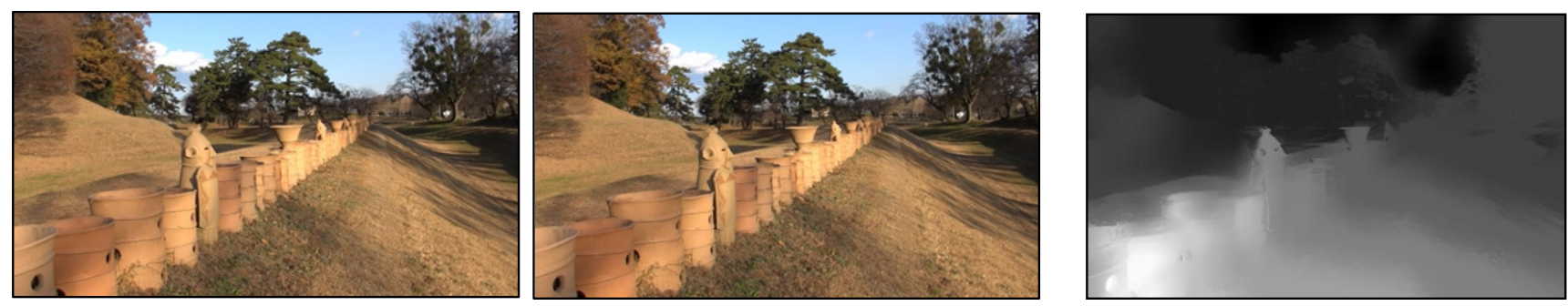

Fig. 8 An example of a scene featured in the educational materials used in this study. Cross fuse for the left pairs (Left: image for right eye; Center: image for left eye: Right: depth map image).

A similar tendency was also seen in the result of Haniwa content (Table 2). In other words, in the 3D only group, there were several questions mainly about the attached objects of the Haniwa, the Haniwa's spatial balance and spatial alignment, which were focused on specific parts (Fig. 7). On the other hand, in the 2D only group, there were questions about the overall positional relationship.

The questions common to both the 3D and 2D groups (Tables 1 and 2) were about basic aspects related to Haniwa, such as many kinds of Haniwa, a face attached to Haniwa, and many Haniwa that were placed. However, having a face on the Haniwa was unique to the educational material that was used and this is not a general feature of the Haniwa.

The results revealed that $3 \mathrm{D}$ educational materials helped them generate novel questions that were not observed in the $2 \mathrm{D}$ observation. An interesting finding was that $3 \mathrm{D}$ educational materials could increase the focus of the students on specific parts in images such as attached objects of the Haniwa, whereas 2D educational materials only helped them view the overall scene (Fig. 7). Also, in 3D view- ing, the students focused on the Haniwa's spatial balance, and spatial alignment. In contrast, in 2D viewing, they focused on the overall characteristics of the Kofun, including the alignment of large areas such as the position of the Kofun and the entrance to the stone chamber and the arranged Haniwa around the Kofun.

The difference of such focus areas suggests that stereoscopic 3D images have the functionality of enabling students to focus their attention on specific parts of the educational materials. This is considered to be related to the disparity of stereoscopic 3D images or the spatial position of reconstructed images placed in front or behind of the display screen. Figure 8 represents an example scene that the students seem to have referred to when viewing stereoscopic 3D images. It is an image that seems to have focused the students' gaze on the order of the Haniwa. The left and center images consist of the stereogram for cross-eyed viewing. The left image is for the right eye and the center image is for the left. The right image is a depth map that can represent different levels of depth and was produced by the 
left and right stereo-pair images. The brightness shows the depth level, which indicates a spatial alignment of the stereo image. The depth map indicates that a row of Haniwa is clearly represented toward the front in comparison to other objects; hence, it is considered that the students focused on this area and then noticed the alignment and gave it considerable thought.

These findings suggest that stereoscopic 3D images could shape the areas of interest that students have in advance. Therefore, the use of $3 \mathrm{D}$ educational materials could be more effective when teachers want to make students focus on important parts from the viewpoint of learning objectives.

From the perspective of inquiry-based learning of history, it is considered important to think deeply about the background of history. The students thought that "because animals were luxury foods" and "because there were animal deities," the figure of the animal was attached to the Haniwa. This can be considered to be a trigger for further exploration into the social positioning of animals of the time. Therefore, 3D images draw students' attention to a part of historical things and geological formations, making it possible to generate questions that are difficult to notice in character and $2 \mathrm{D}$ images materials.

Through the experimental classes, no student reported discomfort or any of the visual fatigues associated with 3D image viewing. Viewing durations were limited to $5 \mathrm{~min}$ per movie, with sufficient time between viewings; however, in general, some individuals with normal vision in both eyes are not good at viewing stereoscopic 3D images. Therefore, despite the strong potential of $3 \mathrm{D}$ images as a learning tool, their use in schools requires careful consideration. Further, there are several uses of displays according to learning design in schools. In addition, it is necessary to pay more attention that students are sometimes required to be closer than normal television viewing distance.

\section{Conclusions}

We examined the advantages of using stereoscopic 3D images for educational purposes in an elementary school class. Comparing two educational materials presented in 2D and $3 \mathrm{D}$, we showed that $3 \mathrm{D}$ viewing could enable students to think in detail about the learning content and thus promote inquiry-based learning. Moreover, the results revealed that 3D educational materials can help students generate novel questions about attached objects of the Haniwa, and Haniwa's spatial balance and spatial alignment. Additionally, 3D educational materials allowed the students to think deeply about the background of history. In concluding the results of the experimental classes, we should note that the educational use of stereoscopic 3D images is worthwhile in that it supports question and hypothesis generation in inquiry-based learning.

In this study, we analyzed only the first two processes out of the eight associated with inquiry-based learning. As a subsequent activity for inquiry-based learning, it is a task for future studies to determine whether we can further promote the learning by combining it with other kinds of 3D educational materials. For example, the study's results show the possibility of applying the same process to other factors of inquiry-based learning, such as investigation (fourth process) and analysis and interpretation (fifth process). In other words, 3D educational materials could possibly be used as an experimental tool to verify a hypothesis. In the classroom, a teacher asks students to think about what and how the order of the Haniwa is formed. The students will make some hypotheses and verify them using 3D images instead of textbooks. They may then notice that the Haniwa are disjointed and prove the hypotheses correct by $3 \mathrm{D}$ observation.

In this study, 3D educational materials were used for a history class in school, and we showed the educational effectiveness although it is a subject that seems to have nothing to do with stereoscopic viewing. As we described earlier, stereoscopic 3D images can directly express depth, concavity, and convexity. Therefore, it is considered to be very effective for education in science, technology, engineering, and mathematics (STEM) as well.

Future studies are required to examine the educational functionality of stereoscopic 3D images in more detail, along with how 3D educational materials can be used for the development of new educational models in school. An appropriate way to use 3D displays in education is also needed to be examined in order to keep students' interests and improve educational effects. In addition, it is necessary for teachers to prepare easy-to-use educational materials with less cognitive load so that they can be practically used in class.

\section{Acknowledgments}

This research was supported by the Japan Society for the Promotion of Science (25560118). We acknowledge the cooperation of the Maebashi Board of Education, which permitted the recording of the Kofun and Haniwa unearthed from an ancient tumulus.

\section{References}

[1] Ministry of Education, Culture, Sports, Science and Technology in Japan, Retrieved Feb. 27th, 2017, from http://www.mext.go.jp/ english/

[2] C. Ware and G. Franck, "Evaluating stereo and motion cues for visualizing information nets in three dimensions," ACM Trans. Graphic., vol.15, no.2, pp.121-140, 1996.

[3] B. Fröhlich, S. Barrass, B. Zehner, J. Plate, and M. Gobel, "Exploring geo-scientific data in virtual environments," Proc. of IEEE: Visualization, vol.99, pp.169-173, 1999.

[4] P.J. Edwards, L.G. Johnson, D.J. Hawkes, M.R. Fenlon, A.J. Strong, and M.J. Gleeson, "Clinical experience and perception in stereo augmented reality surgical navigation," Lecture Notes in Computer Science, vol.3150, pp.369-376, 2004.

[5] R. Zajtchuk and R.M. Satava, "Medical applications of virtual reality," Commun. ACM, vol.40, no.9, pp.63-64, 1997.

[6] Y. Aitsiselmi and N.S. Holliman, "Using mental rotation to evaluate the benefits of stereoscopic displays," Proc. SPIE 7237, 2009.

[7] A. Bamford, "The 3D in education white paper," Technical report, 
International Research Agency, 2011.

[8] T. Shibata, "Utilization of stereoscopic 3D images for social studies class in elementary school," EdMedia, pp.2575-2580, 2014.

[9] T. Shibata, Y. Ishihara, and K. Satou, "Usefulness of stereoscopic 3D images in elementary school classes," SID 2015 DIGEST, vol.46, no.1, pp.126-129, 2015.

[10] Ministry of Education, Culture, Sports, Science and Technology Japan, Shou Gakkou Gakushuu Sidouyouryou Kaisetsu Shakai hen, Toyo kan shuppannsha, 2008.

[11] Ministry of Education, Culture, Sports, Science and Technology Japan, Chu Gakkou Gakushuu Sidouyouryou Kaisetsu Shakai hen, Nihon Bunkyo shuppan, 2008.

[12] Ministry of Education, Culture, Sports, Science and Technology Japan, Koutou Gakkou Gakushuu Sidouyouryou Kaisetsu Chiri Rekishi hen, Kyoiku shuppan, 2010.

[13] T. Bell, D. Urhahne, S. Schanze, and R. Ploetzner, "Collaborative inquiry learning: Models, tools, and challenges," International Journal of Science Education, vol.3, no.1, pp.349-377, 2010.

[14] J. van Drie and C. van Boxtel, "Historical reasoning: Towards a framework for analyzing students' reasoning about the past," Educational Psychology Review, vol.20, no.2, pp.87-110, 2008.

[15] A.P. Nilsen, "Navigating windows into past human minds: A framework of shifting selves in historical perspective taking," J. Learn. Sci., vol.25, pp.372-410, 2016. DOI: 10.1080/10508406.2016. 1160830

[16] J. McCall, Gaming The Past Using Video Games to Teach Secondary History, Routledge, 2011.

[17] D.M. Hoffman, A.R. Grishick, K. Akeley, and M.S. Banks, "Vergence-accommodation conflicts hinder visual performance and cause visual fatigue," Journal of Vision, vol.8, no.3, pp.1-30, 2008. DOI: $10.1167 / 8.3 .33$

[18] T. Shibata, J. Kim, D.M. Hoffman, and M.S. Banks, "The zone of comfort: Predicting visual discomfort with stereo displays," Journal of Vision, vol.11, no.8, pp.1-29, 2011. DOI: 10.1167/11.8.11

[19] M. Lambooij, M. Fortuin, W. IJsselsteijn, B. Evans, and I. Heynderickx, "Measuring visual fatigue and visual discomfort associated with 3-D displays," Journal of the Society for Information Display, vo.18, no.11, pp.931-943, 2010.

[20] K. Ukai and P.A. Howarth, "Visual fatigue caused by viewing stereoscopic motion images: Background, theories, and observations," Displays, vol.29, no.2, pp.106-116, 2008.

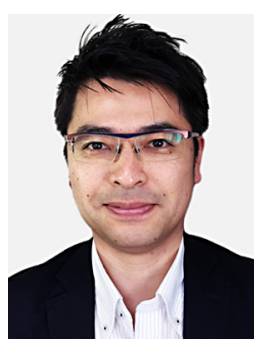

Takashi Shibata received his Ph.D. in Global Information and Telecommunication Studies from Waseda University, Japan, in 2004. Subsequently, he became a research fellow of the Japan Society for the Promotion of Science and a postdoctoral scholar at the University of California, Berkeley. Currently, he is working as an associate professor at the Tokyo University of Social Welfare, Japan. His interests include ergonomics, vision science, and human interface. He is a member of the Applied Vision Sub-committee at the Society for Information Display.

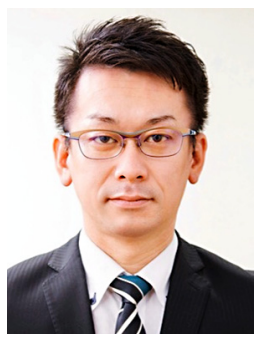

Kazunori Sato received his M.A. in Education from the Joetsu University of Education in 2006. He had been an elementary school teacher at Tokyo Metropolitan from 2006 to 2017. He is currently an assistant professor at the Faculty of Education, Tokoha University, and a Ph.D. student at the Graduate School of Information Sciences, Tohoku University, after enrolling in 2014. He is engaged in research and the development of education in the media and information society.

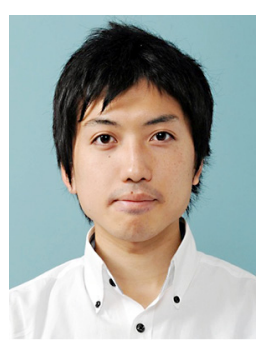

Ryohei Ikejiri received his B.A. in History from the University of Tokyo in 2008 and his Master's and Ph.D. in Interfaculty Initiative in Information Studies from the University of Tokyo in 2010 and 2014, respectively. He is currently an assistant professor at the University of Tokyo. His research interests lie in historical learning, educational technology, and the transfer of learning. 